\title{
LA PROFESIONALIZACIÓN Y LA FORMACIÓN SUPERIOR EN TURISMO
}

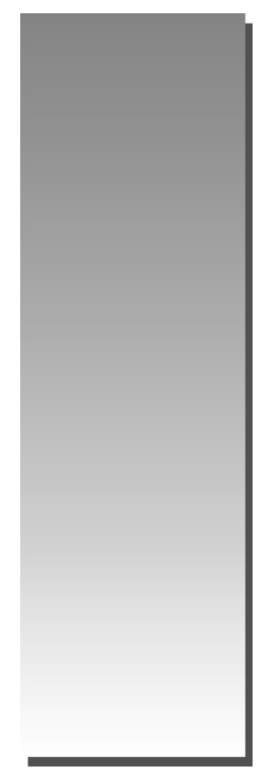

Granados Maguiño Mauro Amaru*

granadosmaurom@gmail.com

\section{RESUMEN}

La actividad turística en el sentido amplio, incluyendo hotelería, gastronomía y ecoturismo, por ser una actividad de servicios, demanda obligatoriamente contar con recursos humanos calificados adecuados a la modernidad y con pleno dominio y aplicación de la tecnología moderna en sus diferentes facetas.

La fuerza laboral de las empresas turísticas en el Perú está compuesta en gran proporción por trabajadores surgidos en el propio puesto ocupacional, es decir, "hechos por la experiencia", principalmente al interior del país.

Estos han sido el gran soporte en la prestación del servicio, que se han ido entrenando, capacitando y calificando de acuerdo a la buena voluntad de las empresas o a la capacidad personal para hacerlo.

Palabras claves: Formación Superior

\section{ABSTRACT}

Tourist activity in the broad sense, including hotel, restaurant and ecotourism, as a service activity, demand compulsory to have adequate skilled human resources to modernity and with full control and application of modern technology in its different facets. The workforce of tourism companies in Peru, is composed in large proportion of workers arising in the post itself occupational ie "made by experience", mainly within the country.

These have been a great support in providing the service, which have been coaching, training and qualifying according to the willingness of companies or staff capacity to do so.

Keywords: Higher education

\footnotetext{
* Director Académico de la Facultad de Ciencias Adminstrativas - UNMSM, Docente de Pregrado y Posgrado de la FCA UNMSM, Magíster en Marketing Turístico y Hotelero, Egresado del Doctorado en Administración.
} 


\section{INTRODUCCIÓN}

A mediados de la década del 60 surge en Lima la formación en turismo en el nivel superior no universitario, que forma profesionales en Administración Turística y en Guía Oficial de Turismo y que comienzan a insertarse en el mercado laboral.

En la década del 80 surge una universidad que ofrece la carrera de turismo en Lima, y comienza a formar profesionales en el área de turismo y hotelería.

En los años posteriores han surgido muchas universidades, tanto en Lima como en el interior del país, que ofertan la carrera de turismo y que, gradualmente, van insertando profesionales en el mercado laboral, desconociéndose aún los grados de inserción de cada una de ellas.

Como se aprecia, hace cuarenta años que se viene formando profesionales de nivel superior, sin embargo, aún subsiste una gran proporción de masa trabajadora que ingresó a la actividad y se hizo en ella "profesional" por la experiencia ganada en el campo. Y a ellos hay que reconocer la fuerza, pujanza y empeño por desarrollarse en la actividad turística.

Precisamente, si hoy en día el turismo ha evolucionado, se ha incrementado, se ha diversificado, presenta preferencias y tendencias que cambian con un dinamismo asombroso, se hace imprescindible establecer mecanismos que permitan profesionalizar a la actividad. Vale decir, que el trabajador que se hizo por la experiencia debe adquirir los conocimientos académicos y teóricos que sustentan los cambios actuales y que permiten ofertar un producto turístico competitivo en todos sus componentes, según el Diccionario de la Lengua Española, en sus 22aㅡ ed., (2002), profesionalizar es:

- Dar carácter de profesión a una actividad.

- Convertir a un aficionado en profesional (una persona ejerce una profesión).

Ello implica, entonces, convertir a los que ingresaron a la actividad sin mayores conocimientos técnicos para desempeñar el puesto ocupacional, en profesionales con conocimientos no sólo en la práctica obtenida por la experiencia, sino complementar con la parte académica formal.

Esto, sin lugar a dudas, contribuirá a mejorar la prestación del servicio turístico, elevando el nivel de calidad del producto turístico y por ende satisfaciendo al consumidor.
La profesionalización no implica solamente la adquisición de conocimientos académicos sino que ello permita que el trabajador se sienta más seguro de sí mismo en su puesto, más calificado y con mayores posibilidades de ascender y mejorar en su nivel ocupacional y en su remuneración personal.

De las 70 universidades, entre nacionales y privadas que se encuentran en funcionamiento a nivel nacional, 36 ofertan la carrera de turismo, hotelería, gastronomía y ecoturismo, es decir, el $51 \%$ del total.

Así, se encuentra que se presentan 22 carreras profesionales, correspondiendo 5 a universidades nacionales, es decir, el 23\% y 17 a universidades privadas, vale decir, el $77 \%$, estas últimas ofrecen mayor variedad.

Actualmente hay una universidad aprobada en Lima que entrará en funcionamiento en el 2010. Y se conoce que al menos hay una organización más que presentará el próximo año su proyecto ante la Conafu.

Del análisis realizado durante la investigación se observa:

- Con referencia a los títulos

La casi totalidad y a nivel nacional, a excepción de uno, es de licenciado, variando en función al matiz que le imprime el plan de estudios.

Se observan 24 denominaciones diferentes, algunos con mención en. No obstante la variedad de títulos, la formación académica es muy similar.

Existe una universidad privada en Lima que oferta dos carreras y otorga doble titulación, licenciado en hotelería y licenciado en administración y licenciado en turismo y licenciado en administración.

Entre los títulos que se otorgan se tiene:

1. Lic. en Turismo

2. Lic. en Turismo y Negocios

3. Lic. en Turismo y Hotelería

4. Lic. en Hotelería y Turismo

5. Lic. en Turismo, Hotelería y Gestión Cultural

6. Lic. en Turismo y Administración

7. Lic. en Administración Turística

8. Lic. en Administración de Turismo

9. Lic. en Administración y Turismo 
10. Lic. en Administración y Negocios Turísticos

11. Lic. en Administración y Servicios Turísticos

12. Lic. en Administración con mención en Turismo

13. Lic. en Administración con mención en Turismo, Hotelería y Gastronomía

14. Lic. en Turismo Sostenible con mención en Gestión y Dirección Turística con mención en Turismo y Desarrollo Local

15. Lic. en Administración de Turismo Sostenible

16. Lic. en Hotelería

17. Lic. en Administración Hotelera
18. Lic. en Administración en Turismo y Hostelería

19. Lic. en Administración de Servicios Hoteleros

20. Lic. en Gastronomía y Gestión de Restaurantes

21. Lic. en Ecoturismo

22. Ingeniero en Ecoturismo

23. Lic. en Hotelería y Licenciado en Administración

24. Lic. en Turismo y Licenciado en Administración

- Con referencia al Plan de Estudios

Todas las carreras ofertadas a nivel nacional se efectúan en 5 años o 10 semestres académicos.

Cuadro No 26. UNIVERSIDADES QUE OFERTAN LA CARRERA DE TURISMO EN LIMA. PLAN DE ESTUDIOS

\begin{tabular}{|c|c|c|c|c|c|c|c|}
\hline & NACIONALES & ESPECIALIDAD & ASIG & CRED & \multicolumn{3}{|c|}{ IDIOMAS } \\
\hline & & & & & ING & FRA & OT \\
\hline 1 & Enrique Guzmán y Valle & & 73 & 210 & 4 & 2 & Q1 \\
\hline \multirow[t]{2}{*}{2} & Federico Villarreal & Ingeniería Ecoturística & 60 & 199 & & & \\
\hline & & Administración Turística & 60 & 199 & & & \\
\hline 3 & Mayor de San Marcos & & 70 & 218 & 8 & & \\
\hline \multirow[t]{2}{*}{4} & José Faustino Sánchez Carrión & & 79 & 251 & 3 & 2 & \\
\hline & PRIVADAS & & & & & & \\
\hline 1 & Alas Peruanas & & 79 & & 6 & 4 & \\
\hline 2 & César Vallejo & & 64 & 213 & & & \\
\hline 3 & Científica del Sur & & 70 & 220 & & & \\
\hline 4 & Piura & & 60 & 240 & & & \\
\hline 5 & Inca Garcilaso de la Vega & & 64 & 180 & & & \\
\hline 6 & Norbert Wiener & & 61 & 208 & & & \\
\hline 7 & Las Américas & & 60 & 200 & & & \\
\hline \multirow[t]{2}{*}{8} & Peruana de Ciencias Aplicadas & Hotelería y Administración & 62 & 165 & & & \\
\hline & & Turismo y Administración & 66 & 165 & & & \\
\hline 9 & San Juan Bautista & & 61 & 61 & & & \\
\hline 10 & Ricardo Palma & & 80 & 200 & 5 & 5 & \\
\hline 11 & Ruiz de Montoya & & 65 & 65 & 5 & & \\
\hline 12 & San Martin de Porres & & 60 & 202 & 3 & & \\
\hline \multirow[t]{3}{*}{13} & San Ignacio de Loyola & Administración en Turismo & 67 & & 7 & & \\
\hline & & Administración Hotelera y & 70 & & 7 & & \\
\hline & & $\begin{array}{l}\text { Gastronomía y Gestión de } \\
\text { Restaurantes }\end{array}$ & 70 & & 7 & & \\
\hline \multicolumn{3}{|c|}{$\mathrm{Q}=$ Quechua } & & & & & \\
\hline \multicolumn{3}{|c|}{$\begin{array}{l}\text { Fuente: Planes de Estudios Universidades } 2008 \\
\text { Elaboración: Propia }\end{array}$} & & & & & \\
\hline
\end{tabular}


El Plan de Estudios está conformado por asignaturas de formación general que generalmente se llevan los dos primeros ciclos y las de especialidad que van con mayor énfasis a partir del tercer ciclo de estudios.

Hay asignaturas de carácter obligatorio y otras de carácter electivos, constituyendo ambas el plan de estudios, pocas universidades incluyen asignaturas electivas.

Se cursan entre 60 y 80 asignaturas durante toda la carrera, presentándose de la siguiente manera:

$\begin{array}{ll}\begin{array}{l}\text { Entre } 60 \text { y } 69 \text { asig- } \\ \text { naturas }\end{array} & \begin{array}{l}60 \% \text { de las universi- } \\ \text { dades }\end{array} \\ \begin{array}{l}\text { Entre } 70 \text { y } 79 \text { asig- } \\ \text { naturas }\end{array} & \begin{array}{l}30 \% \text { de las universi- } \\ \text { dades }\end{array} \\ \begin{array}{ll}\text { De } 80 \text { asignaturas } & \begin{array}{l}10 \% \text { de las universi- } \\ \text { dades }\end{array}\end{array}\end{array}$

Por otra parte, estando organizada la educación universitaria en torno a créditos, estos varían en función a las horas académicas, teóricas y prácticas, de cada asignatura. Así se aprecia que:

En las universidades nacionales varía entre 199 y 251 créditos.

En Las universidades privadas varía entre 165 y 240 créditos.
Las carreras materia de turismo, hotelería y gastronomía, por su propia naturaleza demandan obligatoriamente del conocimiento de, al menos, un idioma extranjero.

En algunas universidades se enseña en mayor proporción el inglés, seguido del francés. Cabe anotar que sólo una universidad enseña la lengua quechua y sólo un semestre.

En algunos casos, cuando se enseña inglés o francés es el técnico, necesario para dominar términos de uso en el ejercicio profesional.

Sólo una universidad ofrece una formación bilinguie español/inglés durante toda la carrera profesional.

En el caso de realizar las prácticas pre profesionales, dependiendo del establecimiento o el lugar donde se efectúe, se exige el dominio de un idioma extranjero, preferentemente el inglés.

$\mathrm{Al}$ respecto, es menester destacar que la mayor parte de universidades no enseña idiomas extranjeros. Explicable el hecho por cuanto en Lima hay muchos centros de enseñanza y los alumnos pueden tomar clases en cualquiera de ellos.

Cuadro № 27. UNIVERSIDADES QUE OFERTAN TURISMO AL INTERIOR DEL PAÍS. PLAN DE ESTUDIOS

\begin{tabular}{|c|l|c|c|c|c|c|}
\hline \multicolumn{1}{|c|}{ NACIONALES } & ASIG & \multicolumn{2}{c|}{ CRED } & \multicolumn{3}{c|}{ IDIOMAS } \\
\hline & & & & ING & FRA & OT \\
\hline 1 & Amazonas & 67 & & 5 & & \\
\hline 2 & Ancash & & & 5 & & \\
\hline 3 & Arequipa & 47 & 303 & 4 & & \\
\hline 4 & Cajamarca & 66 & 216 & 7 & 7 & \\
\hline 5 & Cusco & 82 & 204 & 6 & & Q2 \\
\hline 6 & Ica & 80 & 222.3 & 6 & 2 & A1 \\
\hline 7 & Loreto & 68 & 214 & & & \\
\hline 8 & Madre de Dios & 67 & 216 & & & \\
\hline 9 & Puno & 57 & 232 & & & \\
\hline 10 & San Martín & & & & & \\
\hline 11 & Tumbes & 72 & 184 & I & F & \\
\hline 12 & Huánuco & & 231 & 4 & 5 & \\
\hline & PRIVADAS & 63 & & & & \\
\hline 1 & Arequipa & 66 & 208 & 10 & & \\
\hline 2 & Ancash Chimbote & & & & & IT1 \\
\hline
\end{tabular}




\begin{tabular}{|c|l|c|c|c|c|c|}
\hline 3 & Cusco & 67 & 215 & 10 & & \\
\hline 4 & La Libertad & 59 & 216 & $\mathrm{I}$ & $\mathrm{F}$ & \\
\hline 5 & Lambayeque & 40 & 200 & 3 & & \\
\hline 6 & Puno & & & & & \\
\hline 7 & Tacna & 62 & 236 & & & \\
\hline A $=$ Alemán / Q = Quechua & & & & & \\
\hline TT = Italiano & & & & \\
\hline
\end{tabular}

En el caso del interior del país. Cuadro No 27 El Plan de Estudios está conformado por asignaturas de formación general que generalmente se llevan los dos primeros ciclos y las de especialidad que van con mayor énfasis a partir del tercer ciclo de estudios.

Hay asignaturas de carácter obligatorio y otras de carácter electivos, constituyendo ambas el plan de estudios. Pocas universidades incluyen asignaturas electivas.

Se cursan entre 60 y 82 asignaturas durante toda la carrera, presentándose de la siguiente manera:

Entre 40 y 49 asignaturas

Entre 50 y 59 asignaturas

Entre 60 y 69 asignaturas

De 80 a mas asignaturas

Por otra parte, estando organizada la educación universitaria en torno a créditos, estos varían en función a las horas académicas, teóricas y prácticas, de cada asignatura. Así se aprecia que:

En las universidades nacionales varía entre 184 y 303 créditos.

En Las universidades privadas varía entre 180 y 236 créditos.

Las carreras materia de turismo, hotelería y gastronomía, por su propia naturaleza demandan obligatoriamente del conocimiento de al menos un idioma extranjero.

- Con referencia a las prácticas profesionales

Para una adecuada formación es fundamental la práctica pre profesional, pues pone al alumno en contacto con su futuro puesto ocupacional y con la aplicación de sus conocimientos y valores durante su desempeño.

Todas las universidades contemplan la realización de manera obligatoria de las prácticas pre profesionales en distintas empresas e instituciones relacionadas directa o indirectamente al turismo, hotelería, gastronomía y ecoturismo, tanto a nivel nacional como internacional.

Para ello, suscriben convenios de cooperación interinstitucional que establecen las características y condiciones de las prácticas.

En la mayoría de casos se efectúan en varias oportunidades durante todo el periodo de estudios. Generalmente se efectúan después del cuarto ciclo de estudios. En algunos casos, durante todo el último semestre de estudios.

Algunas universidades han implementado aéreas encargadas de todo el proceso de las prácticas profesionales, desde la búsqueda de la empresa o institución hasta el cierre de la práctica misma.

En otros casos se ha organizado la bolsa de trabajo para brindarle al alumno la posibilidad de la práctica o un empleo.

- Con referencia al perfil profesional

En términos generales se observa que los perfiles profesionales tienen gran similitud, tanto en las carreras de las universidades de Lima como en las del Interior del país.

Hacen referencia:

- Al desarrollo de las capacidades, habilidades y destrezas que debe tener el profesional para desempeñarse como tal.

- Al conocimiento de su propia realidad nacional y de la actividad misma.

- Al conocimiento, identificación y correcta aplicación de técnicas propias de la profesión. 
- A la capacidad de organización, dirección, supervisión y administración de empresas.

- A la capacidad creativa y de innovación que debe demostrar.

- A la capacidad de aportar a través del diseño y desarrollo de proyectos turísticos.

- A la capacidad de transmitir y compartir conocimientos a través de la docencia universitaria y de la investigación.

- Esto indica que se diseña la formación para hacer del profesional un ser humano con valores, que desempeñe una profesión con los más altos niveles de exigencia y competencia en un mercado laboral vasto y complejo.

- Con referencia al campo laboral

En términos generales se observa que el campo laboral es el mismo, tanto en las carreras de las universidades de Lima como en las del interior del país.

Así se tiene:

En empresas de servicios turísticos: establecimientos de hospedaje, restaurantes, cafeterías, discotecas, pubs, karaokes, agencias de viajes y turismo, operadoras de viajes, casinos, cruceros, empresas de transporte turístico, empresas organizadoras de eventos entre otros.

En empresas de recreación: clubes privados y públicos.

En otras empresas: Clínicas, Hospitales.

En empresas asesoras y consultoras en turismo.

En instituciones estatales de turismo: a nivel nacional, regional y local.

En instituciones educativas: docencia en universidades e institutos superiores.

En agencias de viajes, museos, iglesias, restos arqueológicos como guía de turismo.

Hay una universidad en Lima que otorga el título de "Ingeniero en Ecoturismo", indica como posible campo laboral "empresas constructoras a nivel nacional e internacional".

Es importante destacar que si bien es cierto de acuerdo a sus capacidades y conocimientos pueden acceder a un determinado puesto ocupacional, generalmente al ingresar a una empresa lo hacen en un puesto de nivel operativo y, de acuerdo a su desempeño, gradualmente pueden ir ascendiendo de nivel.

\section{Formación superior no universitaria en turismo}

Se han visto ocho institutos superiores tecnológicos localizados en Lima, es decir, aquellos que tienen más presencia en el mercado y realizan mayor actividad, en formación y en capacitación.

La mayor parte de estos ofertan carreras del área de gastronomía y hotelería y, en menor proporción, de turismo. La preferencia se observa en función a las necesidades del mercado laboral.

Así se encuentra que se presentan ocho tipos de carreras profesionales de nivel técnico, lo que corresponde a las instituciones privadas (7) y a la nacional (1).

Del análisis realizado durante la investigación se observa:

- Con referencia a las carreras:

Es preciso destacar que en algunos casos las denominaciones son diferentes pero el contenido es similar con pequeñas variaciones.

Entre las carreras que se ofrece se tiene:

1. Administración de Servicios Hosteleros

2. Administración Hotelera

3. Gastronomía

4. Alta Cocina

5. Pastelería

6. Bartender

7. Administración Turística

8. Guía de Turismo

- Con referencia a los títulos

De las 15 denominaciones observadas se encuentra que, a excepción del guía oficial de Turismo, todos son profesional, profesional técnico y técnico.

Todos los títulos son otorgados por el Ministerio de Educación a nombre de la Nación por cuanto son carreras oficialmente aprobadas por el sector correspondiente. En algunos casos, las propias instituciones educativas otorgan un diploma con una segunda denominación similar. 
En el caso de hotelería en algunos casos combinan con restaurantes y afines

En el caso de cocina se refieren también al arte culinario y gastronomía.

Cabe anotar que algunas de las instituciones educativas consideradas brindan hasta 5 carreras profesionales de nivel técnico.

Muchas de estas instituciones ofrecen paralelamente programas de extensión para carreras cortas de un año y cursos de tres, seis y doce meses. Asimismo, ofertan cursos libres de corta duración, cerca de un mes.

Entre los títulos que se otorgan se tiene:

1. Guía Oficial de Turismo

2. Profesional en Administración Turística

3. Profesional Técnico en Administración de Turismo

4. Profesional en Administración Hotelera

5. Profesional Técnico en Administración de Hoteles y Restaurantes

6. Profesional Técnico en Administración de Hoteles, Restaurantes y Afines

7. Profesional Técnico en Administración de Servicios de Hostelería

8. Profesional en Administración de Negocios Hosteleros

9. Técnico en Administración de Servicios de Hostelería

10. Profesional Técnico en Gastronomía Industrial

11. Profesional Técnico en Gastronomía y Arte Culinario

12. Profesional en Cocina Nacional e Internacional

13. Técnico en Cocina/ Técnico en Alta Cocina
14. Profesional Técnico en Pastelería

15. Técnico en Bar y Coctelería

- Con referencia al plan de estudios

Todas las carreras ofertadas se realizan en tres años. Solo las carreras que ofrece la institución estatal consideran siete ciclos o tres años y medio.

El plan de estudios está conformado por asignaturas de formación general y por las de especialidad que van del tercer al sexto ciclo de estudios, todas las asignaturas son de carácter obligatorio.

Se cursan entre 21 y 71 asignaturas durante toda la carrera, presentándose de la siguiente manera:

Entre 20 y 30 asig- $23 \%$ de las institunaturas ciones.

Entre 31 y 40 asig- $27 \%$ de las institunaturas ciones.

Entre 41 y 50 asig- $27 \%$ de las institunaturas ciones.

Entre 51 y 60 asig- 13\% de las institunaturas ciones.

Entre 61 y 70 asig- 5\% de las institucionaturas nes.

De 71 asignaturas $\quad 5 \%$ de las instituciones.

Las carreras técnicas de turismo, hotelería y gastronomía, por su propia naturaleza, demandan obligatoriamente del conocimiento de al menos un idioma extranjero.

De las ocho instituciones analizadas, seis de ellas enseñan inglés y/o Francés, algunas durante todo el periodo de formación.

En algunas instituciones se enseña en mayor proporción el inglés, seguido del francés. Generalmente es idioma técnico. 
Cuadro № 28 INSTITUTOS SUPERIORES TECNOLÓGICOS QUE OFERTAN TURISMO. PLAN DE ESTUDIOS

\begin{tabular}{|c|c|c|c|c|c|}
\hline & \multirow[t]{2}{*}{ INSTITUTOS } & \multirow[t]{2}{*}{ ASIG } & \multicolumn{3}{|c|}{ IDIOMAS } \\
\hline & & & ING & FRA & OT \\
\hline & CEPEA & & & & \\
\hline 1 & Guías de Turismo & 71 & 6 & 6 & \\
\hline \multirow[t]{2}{*}{2} & Servicios Hosteleros & 66 & & & \\
\hline & CESCA & & & & \\
\hline \multirow[t]{2}{*}{3} & Especialización en Turismo & & & & \\
\hline & EXPRO & & & & \\
\hline 4 & Servicios Hoteleros & 58 & 6 & 3 & \\
\hline 5 & Arte Culinario & 43 & 2 & 2 & \\
\hline \multirow[t]{2}{*}{6} & Alta Cocina & 29 & 2 & 2 & \\
\hline & GASTROTUR & & & & \\
\hline 7 & Empresario de la Gastronomía & 43 & & & \\
\hline 8 & Alta Cocina & 30 & & & \\
\hline \multirow[t]{2}{*}{9} & Profesional Bartender and Manager & 21 & & & \\
\hline & HOSPITALITY MANAGEMENT SCHOOL & & & & \\
\hline 10 & Administración y Gerencia en Hotelería Internacional & 46 & 6 & 3 & \\
\hline \multirow[t]{2}{*}{11} & Alta Cocina & 34 & & 1 & \\
\hline & LE CORDON BLEU & & & & \\
\hline 12 & Cocina & 36 & & 2 & \\
\hline 13 & Gastronomía y Alta Cocina & 54 & & 4 & \\
\hline 14 & Pastelería & 36 & & 4 & \\
\hline 15 & Administración de Hoteles, Restaurantes y Afines & 43 & & & \\
\hline \multirow[t]{2}{*}{16} & Gastronomía Industrial & 23 & & & \\
\hline & SAN IGNACIO DE LOYOLA & & & & \\
\hline \multirow[t]{2}{*}{17} & Administración de Hoteles y Restaurantes & 42 & 6 & & \\
\hline & CENFOTUR & & & & \\
\hline 18 & Administración Turística & 40 & & & \\
\hline 19 & Administración Hotelera & 40 & & & \\
\hline 20 & Guía Oficial de Turismo & 40 & & & \\
\hline
\end{tabular}

En el caso de realizar las prácticas pre profesionales, dependiendo del establecimiento o el lugar donde se efectúen, se exige el dominio de un idioma extranjero, preferentemente el inglés.

Se considera la enseñanza en inglés, por cuanto el nivel ocupacional es fundamentalmente operativo de ahí la necesidad imperiosa de dominar un idioma.

- Con referencia a las prácticas profesionales Considerando que la formación es técnica y el campo ocupacional operativo, la practica pre profesional tiene un papel preponderante.
Todas las instituciones educativas contemplan la realización, de manera obligatoria, de las prácticas pre profesionales en distintas empresas e instituciones relacionadas directa o indirectamente al turismo, hotelería y gastronomía.

Para ello suscriben convenios de cooperación interinstitucional que establecen las características y condiciones de las prácticas.

Generalmente se efectúan después del segundo ciclo de estudios y en algunos casos solo en el último semestre de estudios. 
Algunas instituciones han implementado aéreas encargadas de todo el proceso de las prácticas profesionales, desde la búsqueda de la empresa o institución hasta el cierre de la práctica misma.

En otros casos se ha organizado la Bolsa de trabajo para brindarle al alumno la posibilidad de la práctica o un empleo.

- Con referencia al perfil profesional

En términos generales se observa que los perfiles profesionales tienen gran similitud, diferenciándose según la especialidad, sea turismo, hotelería o gastronomía.

\section{Hacen referencia:}

A la aplicación de técnicas especificas de la profesión.

Al desarrollo de las capacidades, habilidades y destrezas.

A la capacidad de organización, dirección, supervisión y administración de empresas.

A la capacidad creativa y de innovación que deben demostrar.

A la capacidad para generar su propia empresa.

A la capacidad de transmitir y compartir conocimientos a través de la docencia tecnológica e investigación.

Al dominio de un idioma extranjero.

Los rasgos del perfil demuestran que se forma el profesional técnico sobre una solida base técnica complementada por valores como ser humano.

- Con referencia al campo laboral

En términos generales se observa que el campo laboral es el mismo, tanto en las carreras que ofrecen las universidades a nivel nacional como las que ofertan los institutos superiores tecnológicos.

Así se tiene:

En empresas de servicios turísticos: establecimientos de hospedaje, restaurantes, cafeterías, discotecas, pubs, karaokes, agencias de viajes y turismo, operadoras de viajes, casinos, cruceros, empresas de transporte turístico, empresas organizadoras de eventos entre otros.
En empresas de recreación: clubes privados y públicos.

En instituciones estatales de turismo: a nivel nacional, regional y local.

En instituciones educativas: Docencia en institutos superiores.

En agencias de viajes, museos, iglesias, restos arqueológicos como guía de turismo.

Es importante destacar que en el campo laboral convergen, paralelamente, los egresados de universidades y de institutos superiores tecnológicos, que tienen una formación similar, aunque en el caso de los institutos superiores tecnológicos está dirigido fundamentalmente a la función operativa en las áreas pertinentes.

La selección de quien ingrese a ocupar el puesto dependerá no solo de su formación académica sino de la experiencia en prácticas pre profesionales así como de sus características personas y predisposición al empleo y vocación de servicio.

\section{Caracterización de la oferta de formación en turismo}

Como se ha visto previamente, se encuentran muchas similitudes y pocas diferencias entre la formación provista por las universidades y los institutos superiores tecnológicos a nivel nacional. Así se encuentran:

- Titulo a nombre de la nación:

Licenciado a nivel universitario.

Profesional - técnico en institutos superiores tecnológicos.

- Duración de los Estudios:

Entre cinco y tres años.

- Estructura académica:

Asignaturas de formación general y de especialidad.

- Idiomas:

Las universidades nacionales en su mayoría incluyen el idioma Inglés como una asignatura y en algunos casos el Francés.

- Prácticas pre profesionales:

Son de carácter obligatorio en ambos niveles. Se efectúan en el mismo tipo de empresas e instituciones, aunque en algunos casos en diferentes niveles ocupacionales. 
En algunos casos, durante todo un semestre y en otros intercalados.

Hoy en día, el mercado laboral en el sector turismo demanda mucha preparación, se solicita mucha experiencia en esta carrera. No solo las agencias de viajes, por ejemplo, piden personal altamente calificado que domine los sistemas sabre y amadeus, dominio de idiomas también lo hacen los establecimientos de hospedaje, y los restaurantes.

Actualmente hay gran demanda de empleos. En los diarios se puede apreciar la exigencia de las empresas por contar con los mejores counter, administradores, ejecutivas de cuentas, gerentes docentes, como se puede apreciar en los anuncios publicados en el suplemento Aptitus del diario El Comercio entre otros medios.

En dichos pedidos se hace énfasis en:

- Conocimiento técnico del puesto ocupacional

- Dominio de programas informáticos (Procesador de textos y hoja de cálculo, en algunos casos sistemas especializados)

- Dominio de un idioma extranjero, preferentemente ingles

- Experiencia profesional

- Buena presencia

- Disponibilidad inmediata

Hay que precisar que esta solicitud requiere la presentación del Curriculum Vitae y luego de la evaluación, a una entrevista personal y si esta adecuada la persona en los dos aspectos pasa a una entrevista con el psicólogo.

La demanda de puestos ocupacionales está condicionada por seis fenómenos como son:

- Velocidad a la que se producen los cambios;

- Facilidad de acceso a la información;

- Nuevos productos y servicios;

- Globalización de la economía;

- Nuevos mercados;

- Nuevos competidores;

No hay incidencia o preponderancia de uno de ellos, todos en su conjunto se articulan para condicionar un puesto ocupacional.
Los viejos esquemas de producción ya no sirven. Las empresas demandan por lo general profesionales con las siguientes características:

- Sólida formación académica, teórica y práctica en la actividad turística y hotelera a nivel nacional e internacional.

- Amplia cultura general y el dominio de idiomas que le permita interactuar con personas de diversas culturas.

- Liderazgo en el manejo eficaz y eficiente de la actividad empresarial vinculado al turismo y hotelería.

- Capacidad para difundir las más amplias manifestaciones de la cultura peruana.

- Personalidad dinámica y ejecutiva.

- Sólida formación ética y moral que le permita desarrollar sus valores personales.

- Persona con amplia cultura que le permita desenvolverse sin dificultad en el ámbito turístico y hotelero.

- Capacidad de liderazgo.

- Persona con firme predisposición al aprendizaje continuo y a la investigación.

- Capaz de demostrar su espíritu empresarial y de motivar una conciencia turística y hotelera.

- Impulsor del turismo interno y receptivo, protegiendo, conservando y respetando nuestros recursos naturales, la biodiversidad, las expresiones culturales y las tradiciones de cada comunidad, valorando el contacto como un intercambio cultural y orientándolo hacia un desarrollo sustentable.

- Implementador de la calidad total en los servicios turísticos y hoteleros.

- Desarrollo de sus actividades profesionales con respeto a la persona humana.

- Propiciar permanentemente el bienestar social, a través de su eficiente participación profesional.

Asimismo, actualmente el profesional Licenciado en Turismo y Hotelería es solicitado por las empresas de sector turístico, en donde puede desempeñarse, entre otros, como: 
- Gerente y Administrador en empresas turísticas y hoteleras como agencias de viajes, líneas aéreas, restaurantes, hoteles, franquicias, agencias de promoción turística y todo tipo de empresas dedicadas al servicio del turista.

- Responsable de la administración y gestión de nuevos proyectos.

- Asesor y consultor independiente en el área de servicios y negocios turísticos.

- Docencia universitaria e institutos superiores.

- Promotor y ejecutor de proyectos de desarrollo turístico e investigación científica.

- Organizador de congresos y eventos.

- Emprender su propia empresa en este sector.

- Especialista en turismo en las oficinas de turismo de los gobiernos locales y regionales.

- Ocupar puestos directivos en empresas públicas y privadas del sector turístico a nivel nacional o internacional.

Existen aspectos interesantes en la formación turística actual que deben ser consideradas:

- El desarrollo de las nuevas tecnologías aplicadas al sector. Este aspecto es necesario y obligado, para lo cual se están llevando a cabo importantes inversiones en el mundo de la informática aplicada.

- La necesaria especialización de la formación, condicionada por la propia diversidad del sector turístico nacional:

- Es aún un aspecto por desarrollar, para lo cual podría ser una alternativa el desarrollo de estudios de cuarto y quinto, favoreciendo con ello, además de la especialización, la investigación.

- Y el desarrollo de propuestas formativas relacionadas con la calidad y la vinculación entre la docencia y el sector empresarial turístico:

- Aspecto de primera necesidad que se vincula al fomento de las normas de calidad impuestas por el mundo empresarial actual, que agrupa tanto al sector docente como económico. En el caso de los centros de formación en turismo se considera que la mejor estrategia de calidad formativa es la unión indisoluble entre formación teórica y práctica, para lo cual es esencial el desarrollo de programas de prácticas formativas en las empresas del sector.

- La idoneidad de los profesionales de la formación turística.

El modelo de competencia laboral para hotelería y turismo tiene tres componentes:

- Normalización, que consiste en elaborar estándares de calidad del desempeño para todas las funciones y ocupaciones del servicio al turista;

- Evaluación y certificación, que permite verificar concretamente la calidad del desempeño de los trabajadores con relación a los estándares diseñados;

- Formación profesional, que brinda la opción de complementar capacidades a los actuales trabajadores y estructurar itinerarios educativos adecuados a los nuevos profesionales del turismo, en concordancia con las demandas del mercado turístico;

- Se trata de un sistema desarrollado en varios países, con resultados óptimos y satisfactorios. El objetivo es beneficiar a todos los actores de la actividad hotelera y turística, desde el empresario hasta el trabajador, dándoles la oportunidad de tomar decisiones acertadas e incorporarse a un sistema que garantiza su alto nivel de calidad en el servicio. Pero sobre todo busca mejorar la imagen turística del Perú, porque al hacer posible la mejora de calidad del servicio a niveles internacionales, provocará el despliegue del más eficiente instrumento de marketing que existe: la recomendación del turista satisfecho.

Las principales características de la formación profesional son las siguientes:

- Desempeño profesional competente

Formar personas calificadas que respondan a las necesidades de un trabajo productivo de calidad, desarrollando capacidades para el manejo técnico de las diversas áreas del sector hotelero y turístico con eficiencia y responsabilidad, incorporando aspectos actitudinales e instrumentales que permitan "aprender a 
aprender" y "aprender a trabajar" a lo largo de la vida productiva. Este desempeño competente está descrito y basado en las Normas de Competencia Laboral.

- Mayor movilidad y adaptabilidad laboral

Formar personas que sean capaces de responder a los distintos requerimientos de calificación que se les presente a lo largo de su vida profesional; personas capaces de actualizarse permanentemente de manera autónoma, adecuarse y responder asertivamente a los cambios del mercado laboral.

- Flexibilidad de la formación

Brindar a los distintos beneficiarios de la formación profesional la posibilidad de adquirir competencias laborales organizadas en módulos educativos, permitiendo tener opciones de empleabilidad durante su formación y mayor facilidad de reinsertarse en el sistema formativo, si este fuera el caso.

\subsection{Objetivos de la formación profesional}

Entre los principales objetivos están:

- Calificar los recursos humanos del sector hotelero, gastronómico y turístico, brindando una formación de calidad pertinente, con capacidad de respuesta a las necesidades de individuos y empresas, creando hábitos y actitudes que permitan incorporarse en procesos de formación permanente.

- Articular la formación profesional con el mundo del trabajo, incentivando el desarrollo de una mentalidad productiva y una cultura de trabajo acordes a las demandas actuales y futuras del sector.

- Formar personas capaces de emprender iniciativas empresariales competitivas en el sector, que tengan trascendencia local y nacional que beneficien el desarrollo cualitativo y cuantitativo de recursos humanos de calidad.

- Brindar diversas opciones y grados de formación y capacitación técnica de acuerdo a las demandas definidas por el sector, así como una estructura flexible que permita a los beneficiarios diversos itinerarios con entradas y salidas del sistema.

- Estimular las iniciativas de innovación o adecuación tecnológica que tengan repercusión en la productividad del sector.

Es importante destacar que lo que se quiere lograr es una formación de calidad e integral donde converjan las potencialidades y requerimientos personales, profesionales y laborales.

\subsection{Las áreas y grados de la formación profesional}

La formación profesional en hotelería y Turismo se organiza en grados de formación, que describen los diversos niveles educativos definidos de acuerdo a la demanda del sector.

Además, se organiza en áreas de acuerdo a los procesos productivos de servicios del sector, que se basan en los resultados de la Normalización de Competencia Laboral.

En general, la organización se sintetiza en el siguiente esquema:

Cuadro No. 29 La Acreditación Profesional

\begin{tabular}{|c|c|c|c|c|}
\hline Escuelas & \multicolumn{2}{|c|}{ Hotelería } & \multicolumn{2}{|c|}{ Turismo } \\
\hline $\begin{array}{c}\text { Áreas de } \\
\text { Competencia } \\
\text { Laboral: } \\
\end{array}$ & Hospedaje & $\begin{array}{c}\text { Alimentos y } \\
\text { Bebidas }\end{array}$ & $\begin{array}{c}\text { Comercialización } \\
\text { y Operaciones } \\
\text { Turísticas } \\
\end{array}$ & Transporte \\
\hline \multirow{4}{*}{$\begin{array}{c}\text { Formación de } \\
\text { Grados }\end{array}$} & \multicolumn{4}{|c|}{ Grado básico } \\
\hline & \multicolumn{4}{|c|}{ Grado técnico } \\
\hline & \multicolumn{4}{|c|}{ Grado profesional } \\
\hline & \multicolumn{4}{|c|}{ Segunda especialización } \\
\hline
\end{tabular}


- Las áreas de la formación profesional

Las áreas de la formación profesional se basan en los resultados de la Normalización de Competencia Laboral, que fundamenta su organización en las áreas de competencia laboral definidas para el sector hotelero y turístico nacional.

Un área es un conjunto integrado de funciones que conducen a la producción de servicios hoteleros y turísticos.

En el presente caso, se identifican dos subsectores (hotelería y turismo) y cuatro áreas (hospedaje, alimentos y bebidas, comercialización y operaciones turísticas, transporte).

La organización en áreas permite el ordenamiento temático de la oferta, respondiendo de manera integral a la demanda de formación. Las ventajas son las siguientes:

Oferta ordenada de acuerdo a las funciones reales de las áreas de competencia laboral.

Atención a diversos y heterogéneos criterios de demanda, tipos de empresas y modalidades de formación o capacitación.

Coherencia para la actualización y renovación de la oferta formativa y de capacitación.

Ordenamiento administrativo de los centros de formación.

El presente esquema sintetiza la organización por áreas de competencia laboral propuesta para los centros de formación:

\section{Grafico No 16 ÁREAS DE COMPETENCIA LABORAL}

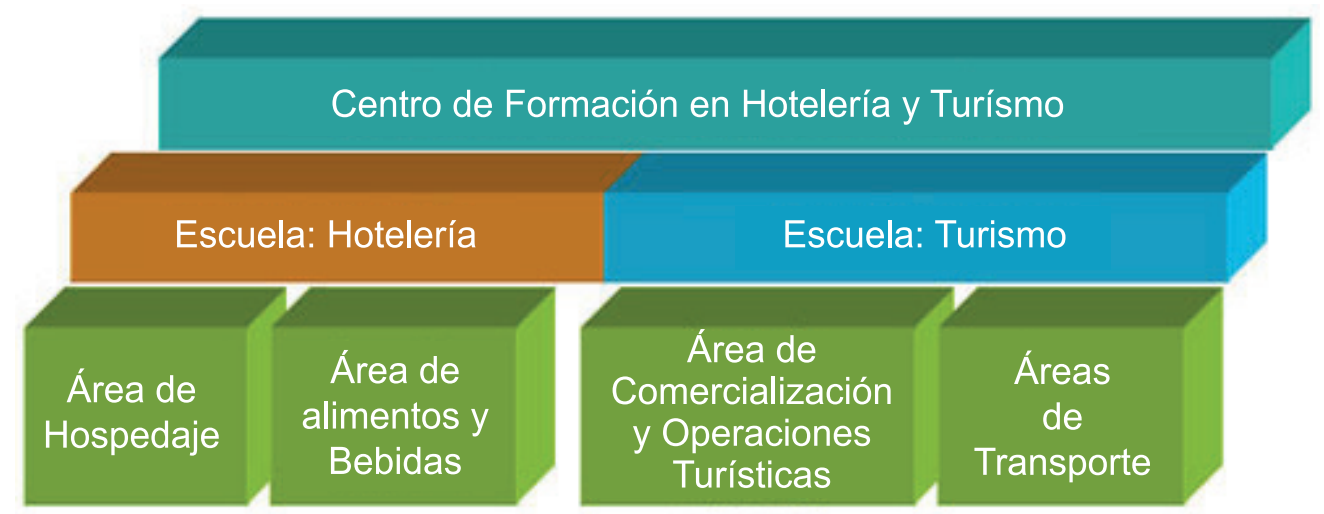

- Los Grados de la formación profesional

El nuevo modelo de formación profesional propone cuatro grados de formación en hotelería y turismo. Estos grados son definidos en función a las características de la demanda del sector, recogidas y organizadas en el Componente de Normalización de Competencias Laborales.
En la nueva concepción de la formación profesional, existe una relación entre los Niveles de competencia laboral (demanda del sector plasmada en la Normalización de Competencias Laborales) y los grados de formación (organización del tipo de formación de acuerdo a la demanda recogida en la Normalización).

Esta relación se esquematiza de la siguiente forma:

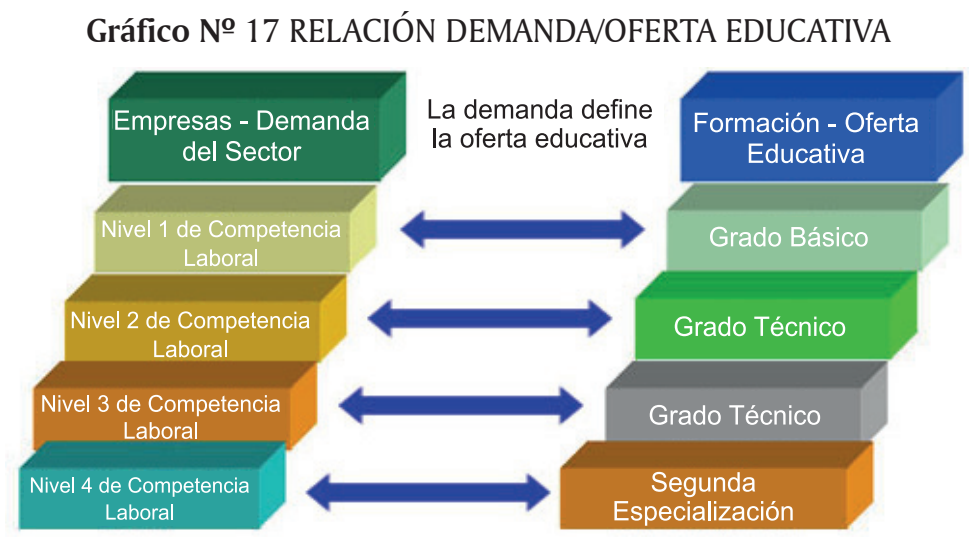


En general, podemos definir los Grados de Formación Profesional de la siguiente manera:

Grado Básico: Formación orientada a ocupaciones con funciones puntuales, de poca complejidad técnica. Supone el desarrollo de capacidades fundamentales de atención y calidad en el servicio.

Grado Técnico: Formación orientada a ocupaciones operativas, con funciones caracterizadas por la ejecución de procesos de producción de servicios, con niveles de decisión técnica y de responsabilidad en su área de trabajo. Supone además el desarrollo de trabajo en redes laborales, capacidades de coordinación y desarrollo de actitudes de servicio acordes a las demandas que su función exige.

Grado Profesional: Formación orientada a ocupaciones operativas que requieren de capacidades de organización y supervisión de áreas de trabajo y redes laborales, con plena autonomía en las decisiones técnicas y de responsabilidad en su área de trabajo.

Segunda Especialización: Formación orientada a la especialización en determinadas ocupaciones y el desarrollo de capacidades en las áreas de gestión y organización de empresas. Esta opción presupone una formación profesional previa o competencias adquiridas en el mundo laboral.

Como profesionales deberán cumplir con lo siguiente:

- Aportar al desarrollo del país, estructurando estrategias que incentiven la actividad turística y hotelera, teniendo en cuenta la realidad socio económica, política y cultural.

- Identificar, analizar y solucionar creativamente los problemas que en su actividad profesional se puedan presentar.

- Investigar y conocer la realidad de la actividad turística y hotelera, para proponer planes integrales, que en el mediano plazo conviertan al país, en un centro turístico de importancia a nivel mundial.

- Actuar profesional y responsablemente en todos los niveles de la actividad turística y hotelera.

- Crear modelos de gestión, capaces de aprovechar las diversas ventajas comparativas que tiene el país para el desarrollo integral del turismo y la hotelería.

- Liderar las empresas a su cargo a base de una visión amplia de la actividad turística y hotelera.

En la actualidad existen una serie de oportunidades y circunstancias que el Perú debe aprovechar para un próspero desarrollo de su industria gastronómica.

Con la entrada de los TLC en nuestro país, en definitiva se presenta una mayor oportunidad para el incremento del ingreso de turistas, lo que beneficia y ofrece oportunidades importantes para los hoteles, restaurantes y demás servicios turísticos.

En el futuro un profesional tiene principalmente que:

- Ser capaz de aplicar el proceso administrativo en cualquiera de las áreas funcionales, mercadotecnia, producción, finanzas y recursos humanos, así como tomar decisiones basado en la información obtenida de todas las áreas fundamentales y de los medios de control.

- Coordinar los elementos materiales técnicos y humanos para lograr los objetivos con máxima eficiencia.

- Formular diagnósticos económicos sociales de las organizaciones y asimilar el espíritu de colaboración, solidaridad y servicio como filosofía en bien de la comunidad.

- Tener una visión agresiva de conjunto que le permita al profesional ir conquistando los niveles más altos dentro de este sector creciente del mercado de servicios.

- El profesional managerial del futuro habrá de ser más coacher y líder que jefe / controller en el sentido clásico. Es decir, habrá de ejercer más su autoridad y menos el poder otorgado. Habrá de orientarse más en facilitar la tarea de los demás y no tanto en mandar y controlar. Se impondrá la transparencia versus la opacidad de la anticuada creencia de que la información es poder. Primará la comunicación.

- Saber usar más su inteligencia emocional, buscar más el compromiso que la sola adhesión, dedicar más esfuerzos a la generación de deseo que a dar órdenes de obligaciones a cumplir.

- Saber escuchar más y mejor. 


\section{La profesionalización como objetivo y responsabilidad}

Teniendo en cuenta que el turismo, como actividad de servicios debe de tener mucho cuidado en cuanto a los recursos humanos, hay que promover con carácter obligatorio la Profesionalización de la actividad.

Y esa tarea constituye una responsabilidad y un compromiso. Responsabilidad en todos los que producen el producto turístico y compromiso en quienes dirigen y manejan el sector turístico.

Si la educación es un derecho del ser humano, manifiesta esta en cualquiera de sus niveles, etapas o formas, la formación y capacitación debe ser un hecho continuo, permanente y organizado.

La variedad de empresas que conforman el sector turismo demanda de la organización y propuesta específica de acciones coordinadas y sostenidas dirigidas a elevar y mejorar las condiciones de la calidad de la prestación del servicio turístico.

Si la Ley General de Turismo establece que el sector correspondiente tiene a su cargo la formulación de los lineamientos de política que promuevan el desarrollo de los recursos humanos, este debe actuar y cumplir oportunamente.

En ese contexto, como parte del Plan Nacional de Calidad Turística, CALTUR y en concordancia con el Objetivo especifico 1 que refiere que los recursos humanos competentes constituyen la base de la gestión de la oferta turística a todo nivel, se podría incluir un Programa Especial denominado "Profesionalizar es Avanzar".

A continuación se bosqueja el programa, en cuanto a la organización, al levantamiento de la encuesta y de la capacitación.

De Organización:

1. Proponer un Programa Especial denominado "Profesionalizar es Avanzar"

Ente responsable: Ministerio de Comercio Exterior y Turismo

Ente financiero: PROMPERU / Organismo Internacional Financiero

Objetivo general: Contribuir a elevar el nivel de calidad del producto turístico

Objetivos específicos:

a) Complementar la formación "por experiencia” de los trabajadores del sector con formación académica especializada y humana. b) Dotar de los nuevos paradigmas, avances tecnológicos, técnicos y herramientas para la gestión administrativa y operativa de las empresas turísticas.

c) Contribuir a mejorar la posición jerárquica de los trabajadores dentro de las empresas turísticas.

d) Contribuir a su superación personal.

Cobertura geográfica: A nivel nacional.

Cobertura empresarial: Empresas de servicios turísticos.

Ámbito Laboral: todos los trabajadores de las empresas turísticas.

Nivel académico: Universitario y Superior No Universitario.

Duración: 5 años, implementado por etapas.

2. Mediante resolución ministerial, declararlo de prioridad sectorial.

3. Mediante resolución ministerial nombrar un comité de gestión, conformado por un representante del Mincetur, quien lo presidirá, un Representante de Canatur, Ahora, APAVIT y APOTURE y un representante del Cenfotur. Tendría por finalidad la programación, gestión y supervisión del programa.

4. Elaboración de los términos de referencia para convocar a un concurso público para realizar una encuesta.

5. Convocar a concurso público, mediante licitación para realizar la encuesta que tendría por finalidad determinar el real nivel de calificación de los trabajadores de la actividad turística a nivel nacional.

6. Comunicar a las DIRCETUR, a nivel nacional las características del programa para asegurar su participación y apoyo.

7. Convocar a los gremios y empresas de servicios turísticos a nivel nacional a participar y apoyar el Programa, difundiendo sus beneficios.

Del Levantamiento de la encuesta

1. Selección y otorgamiento de la buena pro a la empresa ganadora.

2. Levantamiento de la Encuesta a Nivel Nacional: se debería aplicar por grupos de destinos turísticos. La propuesta de ciudades la determinará el Comité de Gestión conjuntamente con la 
Consultora. Se recomienda, al inicio seleccionar destinos que oferten un producto turístico en grado de gran avance para consolidar el nivel de calidad del producto turístico.

3. Del Resultado de la Encuesta. Una vez concluido el proceso del levantamiento de la Encuesta, tabulación y análisis, los resultados servirán de base para el Diseño del Programa de Profesionalización.

\section{De la Capacitación}

Una vez obtenidos los resultados de la encuesta, deberá diseñarse un programa de "Capacitación hacia la Profesionalización"

En esta etapa, de tipo académica, deberán participar en el caso del departamento de Lima, dos entidades educativas.

Una Universidad (UNMSM) para la acción correspondiente al nivel superior directivo y ejecutivo y el Cenfotur, como entidad estatal correspondiente al nivel técnico de mando medio y básico.

La Universidad particular tendría que ofertar las carreras de Turismo, Hotelería y Gastronomía, debiendo tener promoción ya egresada.

El Cenfotur, por tener las carreras de Turismo, Hotelería y Guía Oficial de Turismo, así como por contar con filiales al interior del país que podrían servir juntas con las Dircetur de soporte durante todo el proceso.

Es importante señalar que al término de los estudios recibirán un Diploma que acredite su formación y capacitación, emitido a nombre del MINCETUR y/o de la Universidad o del Cenfotur.

Paralelamente, la empresa de servicios turísticos donde el trabajador se desempeñe, recibirá un Reconocimiento del MINCETUR o Gobierno Regional por su apoyo al desarrollo del Programa.

El MINCETUR, podría dentro del Programa Caltur, establecer un Sello A la Calidad, el cual se entregaría a aquellas empresas turísticas que acreditaran fehacientemente que el $80 \%$ de sus trabajadores en todos los niveles laborales ha recibido formación o capacitación de manera formal y mejor aún si han participado en el Programa "Profesionalizar es Avanzar".
Es importante señalar que el desarrollo del Programa debe efectuarse gradualmente durante cinco años de manera de lograr una cobertura del $100 \%$ del mercado laboral.

Este programa que sería concebido, programado y ejecutado bajo la responsabilidad del MINCETUR, con participación de otras organizaciones públicas y privadas estaría permanentemente supervisado por el Comité de Gestión que tiene las representaciones pertinentes.

El Programa mencionado, actuaría como un gran paraguas sobre los trabajadores de la actividad, si se quiere darle una cobertura amplia y lograr los niveles de eficiencia que el trabajador debe poseer. Sin lugar a dudas requiere de dos elementos sustanciales. La decisión del MINCETUR para lograr su aprobación y conseguir su financiamiento, la participación activa de las empresas turísticas a través de sus trabajadores y de las entidades educativas como entes ejecutores del programa. De la confluencia de decisiones, esfuerzos y compromisos de los tres será posible concretar no un anhelo sino una imperiosa necesidad: La profesionalizacion de los trabajadores del sector turismo.

\section{CONCLUSIONES}

1. La Profesionalización en la actividad turística es fundamental para fortalecer la misma y aún no se han definido políticas ni acciones para lograrlo. Esto implica el trabajo organizado y coordinado del estado y del sector privado, tanto gremios como empresarios, que aún no se ha hecho.

2. La Profesionalización está relacionada con la formación y capacitación de tal manera de proveer al profesional "formado en la experiencia" de los conocimientos, conceptos y formación académica necesarios combinándolo con la vocación, entusiasmo y experiencia en el trabajo.

3. La Profesionalización de la actividad turística permitirá elevar el nivel de calidad de los productos turísticos pudiendo así satisfacer las necesidades y preferencias de la demanda.

4. La Profesionalización debe ser un objetivo pero a la vez una responsabilidad y un compromiso. 


\section{LITERATURA CITADA}

Libros:

- ALBARRACIN, Daniel

- 2004 La industria turística y las relaciones laborales, empleo turístico en España. Fundación Cirem Madrid

- ALIDE y BUFETE CONSULTA

- 2009 El turismo: políticas de desarrollo y potencial de inversiones para la economía de América Latina y el Caribe Curazao

- ARELLANO INVESTIGACIÓN DE MARKETING

- 2009 Dimensionamiento integral del aporte de la gastronomía y la industria alimentaria peruana Lima

- BCP Securities

- 2008 Perspectivas financieras y económicas para la América Latina s.l.

- CERTO, Samuel

- 2003 Administración moderna 8a edición Editorial Pearson Santa Fe de Bogotá

- CONSEJO MUNDIAL DE VIAJES Y TURISMO Y LA FUNDACIÓN PRO - EDUCACIÓN EN TURISMO

- 2005 México empleo para el milenio México, D.F.

- CHACALTANA, Juan 2002 Indicadores dinámicos del empleo Lima

- CHACAlTANA, Juan 2002 Restricciones a la generación de empleo en el sector turístico OIT Lima

- CHACALTANA, Juan 1999 El turismo en el Perú: perspectivas de crecimiento y generación de Empleo, OIT Lima

- ECOGOALS CONSULTING + MANAGEMENT

- 2008 Plan Nacional de Calidad Turística del Perú. MINCETUR Lima

- FONDO MONETARIO INTERNACIONAL

- 2008 Perspectivas económicas mundiales Washington, D.C.

- FORO ECONÓMICO MUNDIAL
- 2009 Competitividad turística

- GALLEGO, Jesús Felipe 1999 Los nuevos perfiles profesionales de los directivos hoteleros, Editorial Alción, Madrid

- HAYA DE LA TORRE DE LA ROSA, Raúl 2005 Estudio sobre la situación de la educación superior tecnológica en el Perú. Informe final FORTEPE, Lima

- HERNANDEZ FERNÁNDEZ Baptista 1998 Metodología de la Investigación Editorial McGraw Hill

- INSTITUTO NACIONAL DE ESTADÍSTICA E INFORMÁTICA 2009 Encuesta permanente de empleo Lima

- INSTITUTO NACIONAL DE ESTADISTICA E INFORMATICA 2009 Lineamientos en el mercado laboral de Lima Metropolitana. Informe Técnico № 6 Lima

\section{REVISTAS}

- ARAMBURÚ, Carlos y MENDOZA, María Isabel

- 2003 "La población peruana, reto y perspectivas"

- Economía y Sociedad 50 CIES (Nov. 2003):45-54

- BEGAZO VILLANUEVA, José Domingo 2007 "Competitividad y percepción del servicio turístico peruano, estudio de investigación 2005" Gestión en el Tercer Milenio, No. 19 F.CC.AA. UNMSM (Julio 2007):45-52

- BORRAS, MATEO 1999 "El nuevo rol de los trabajadores a través de la visión del directivo de RRHH".

- Alta Dirección № 208 (Noviembre - Diciembre 1999): 480

- FRANGIALLY, Francesco 1998 "Mirando la bola de cristal" Revista Valenciana d' Estudis Autonomics № 25 (Octubre 1998): 7-12

- MEYER, Daniel y GARCÍA, Renato 2008 "Tendencias del turismo en el siglo XXI" XVIII Congreso Panamericano de Educación Turística (Noviembre 2008) 
- "OMT: o caminamos juntos o nos ahorcaran por separado" 2009 La agencias de Viajes Chile No. 430 año XVI (Abril 2009): 3

- ORGANIZACIÓN MUNDIAL DEL TURISMO 2008 Barómetro OMT del turismo mundial (Junio 2008)

- ORGANIZACIÓN MUNDIAL DEL TURISMO 2009 Barómetro OMT del turismo mundial (Junio 2009)

- VALENZUELA RAMÍREZ, Irina E. 2009 "Perspectivas el sector turismo en un contexto de crisis internacional" Coyuntura No 22: PUCP, (Febrero 2009): 7-12

- VARGAS, Fernando 2002 "4 afirmaciones en torno a la certificación - Todas Falsas - Boletín Cinterfor № 153 - OIT (2002) :85-112

\section{PAGINAS WEB}

www.apega.com.pe www.arellanomarketing.com www.badatur.com.pe www.canatur.org www.consorcio.edu.pe www.checkperu.org www.elcomercioperu.com.pe www.elprofesional.com.pe www.gestion.pe www.grade.org.pe www.minam.gob.pe www.mincetur.gob.pe www.mindedu.gob.pe www.observatorioturisticodelperu.com www.oit.org.pe www.pucp.edu.pe www.sectur.gob.mx www.sociedadhotelesdelperu.org www.tnews.com.pe www.ttc.org www.wikipedia.org 